\title{
CERAMBYCIDAE (COLEOPTERA) DA COLÔMBIA. III. CERAMBYCINAE COM OLHOS FINAMENTE GRANULADOS
}

\author{
Maria Helena M. Galileo, ${ }^{1,3}$ \\ Ubirajara R. Martins ${ }^{2,3}$
}

\begin{abstract}
CERAMBYCIDAE (COLEOPTERA) OF COLOMBIA. III. CERAMBYCINAE WITH FINELY GRANULATED EYES. New species described: Chrysoprasis pilosa from Huila. New records for Colombian Cerambycidae fauna: Tillomorphini, Euderces posticus (Pascoe, 1866); Heteropsini, Alloesia chlorophana Chevrolat, 1862; Monnecles apollinarii (Gounelle, 1913); Trachyderini, Ancylocerina, Ceralocyna variegata Monné \& Napp, 1999; Trachyderini, Trachyderina, Crioprosopus cacicus Bates, 1885; Ancylosternus m. morio (Fabricius, 1787); Ozodera callidioides Dupont, 1840; Trachyderes hermani Huedepohl, 1985.
\end{abstract}

KEYWORDS. Heteropsini, new records, new species, Tillomorphini, Trachyderini.

\section{INTRODUÇÃO}

Uma relação dos Cerambycidae mencionados para a Colômbia foi publicada por Martínez (2000). Martins \& Galileo (2002a, b), com base em material proveniente da Colômbia, trataram das tribos Eburiini e Ibidionini. Em continuidade à série de artigos que objetiva acrescentar novas ocorrências e adicionar novos táxons à fauna colombiana, enfoca-se as espécies com olhos finamente granulados especialmente das tribos Heteropsini, Tillomorphini e Trachyderini (Ancylocerina e Trachyderina).

O material foi enviado por Claudia Martínez, Instituto de Investigaciones de Recursos Biológicos "Alexander von Humboldt", Villa de Leyva (IAHC) e pertence à Facultad de Agronomia, Universidad Nacional de Colombia, Santafé de Bogotá (UNAB) e à Coleção Gilberto Mendoza, Santafé de Bogotá (GMIC). Parte desse material foi retido para o Museu de Zoologia, Universidade de São Paulo (MZSP).

\section{Tillomorphini \\ Euderces posticus (Pascoe, 1866)}

Apilocera posticus Pascoe, 1866:295, est. 20, fig. 4.

Euderces postica; LACORDAIRE, 1869:90; MonNÉ, 1993:70 (cat.).

Eplophorus posticus; Blackwelder, 1946:583 (cat.).

Euderces posticus; Giesbert \& CheMsaK, 1997:263, figs. 2, 42; Martínez, 2000:91.

A cor do tegumento nesta espécie varia de avermelhado até preto. No holótipo,

1. Museu de Ciências Naturais, Fundação Zoobotânica do Rio Grande do Sul, Caixa Postal 1188, Porto Alegre RS, 90001-970, Brasil.

2. Museu de Zoologia, Universidade de São Paulo, Caixa Postal 42694, São Paulo SP, 04299-970, Brasil.

3. Bolsista do CNPq. 
figurado por PASCOE (1866: 295, est. 20, fig. 4), o tegumento é "brownish-red". Um dos exemplares ora examinado tem cabeça, antenas, protórax e escutelo avermelhados e os élitros pretos; no outro exemplar apenas a cabeça, a base das antenas e a orla anterior do protórax são avermelhadas, o restante do corpo é preto. Euderces posticus caracterizase pelo espinho no lado interno do ápice do antenômero IV que, com raras exceções, está ausente nas outras espécies.

Descrita originalmente de Santa Marta, Magdalena, foi arrolada por MARTínez (2000) para Bolívar e Magdalena.

Material examinado. COLÔMBIA, Magdalena: PNN (Parque Nacional Natural) Tayrona (Pueblito, $11^{\circ} 20^{\prime} \mathrm{N}, 7^{\circ} 02^{\prime} \mathrm{W}, 225 \mathrm{~m}$ ), '- 13-28.VI.2000, R. Henriquez col., armadilha Malaise (IAHC); (Cañaveral, $11^{\circ} 20^{\prime} \mathrm{N}, 74^{\circ} 02^{\prime} \mathrm{W}, 30 \mathrm{~m}$ ), '-, 2-22.I.2001, R. Henriquez col., armadilha Malaise (MZSP).

\section{Heteropsini \\ Alloesia chlorophana (Chevrolat, 1862)}

Alloesia chlorophana Chevrolat, 1862:762; Monné, 1994a:23 (cat.).

Alloesia chlorophana var. aeneipenne Chevrolat, 1862:762; MonnÉ, 1994a:23 (cat.).

Alloesia chlorophana var. hilare Chevrolat, 1862:762; Monné, 1994a:23 (cat.).

Alloesia chlorophana var. nitidepenne Chevrolat, 1862:762; Monné, 1994a:23 (cat.); Martínez, 2000:87.

Esta espécie parece ser comum na Colômbia e MARTíneZ (2000) assinalou-a para Antioquia, Boyacá e Cundinamarca.

Material examinado. COLÔMBIA, Tolima: Herveo $\left(5^{\circ} 04^{\prime} 59^{\prime \prime} \mathrm{N}, 75^{\circ} 10^{\prime} 46^{\prime \prime} \mathrm{W}\right),{ }^{\prime}$, 23.XII.1971, A. Quintero col. (UNAB).

\section{Monnecles apollinarii (Gounelle, 1913)}

Heterops ? apollinarii Gounelle, 1913:420; Monné, 1994a:34 (cat.); Martínez, 2000:88.

Monnecles apollinarii; NAPP \& SANTos, 1999:283, fig. 1.

Originalmente descrita com base em 8 síntipos (5 machos e 3 fêmeas ) coletados por W. Fritsche na montanha La Garita. MARTínez (2000) registrou-a para Bolívar e Norte de Santander.

Material examinado. COLÔMBIA, Norte de Santander: Villa del Rosario $\left(7^{\circ} 50^{\prime} 02^{\prime \prime N}\right.$, 72²8’29" W, $431 \mathrm{~m}$ ), ${ }^{\circ}, '-$, 05.IV.1971, P. Soto col. "al vuelo" (UNAB, MZSP).

\section{Chrysoprasis pilosa sp. nov.}

(Fig. 1)

Fêmea. Colorido geral verde-metálico exceto antenas, metade basal dos fêmures, tíbias e tarsos, pretos; urosternitos vermelhos. Cabeça densamente pontuada, sem microescultura. Fronte mais larga do que longa. Parte centro-superior do clípeo losangular e lisa. Área malar tão longa quanto o lobo inferior do olho. Antenas pretas; escapo pontuado com leve brilho verde-metálico; atingem o ápice do élitro no meio do antenômero XI; antenômeros distais expandidos no lado externo; antenômeros III-V com espinho apical; antenômero VI com espinho apical diminuto. Protórax (fig. 1) mais largo do que longo; lados arredondados com a maior largura no meio. Pronoto com tegumento brilhante, densamente alveolado, sem microescultura; pilosidade amarelo-dourada, bem evidente 

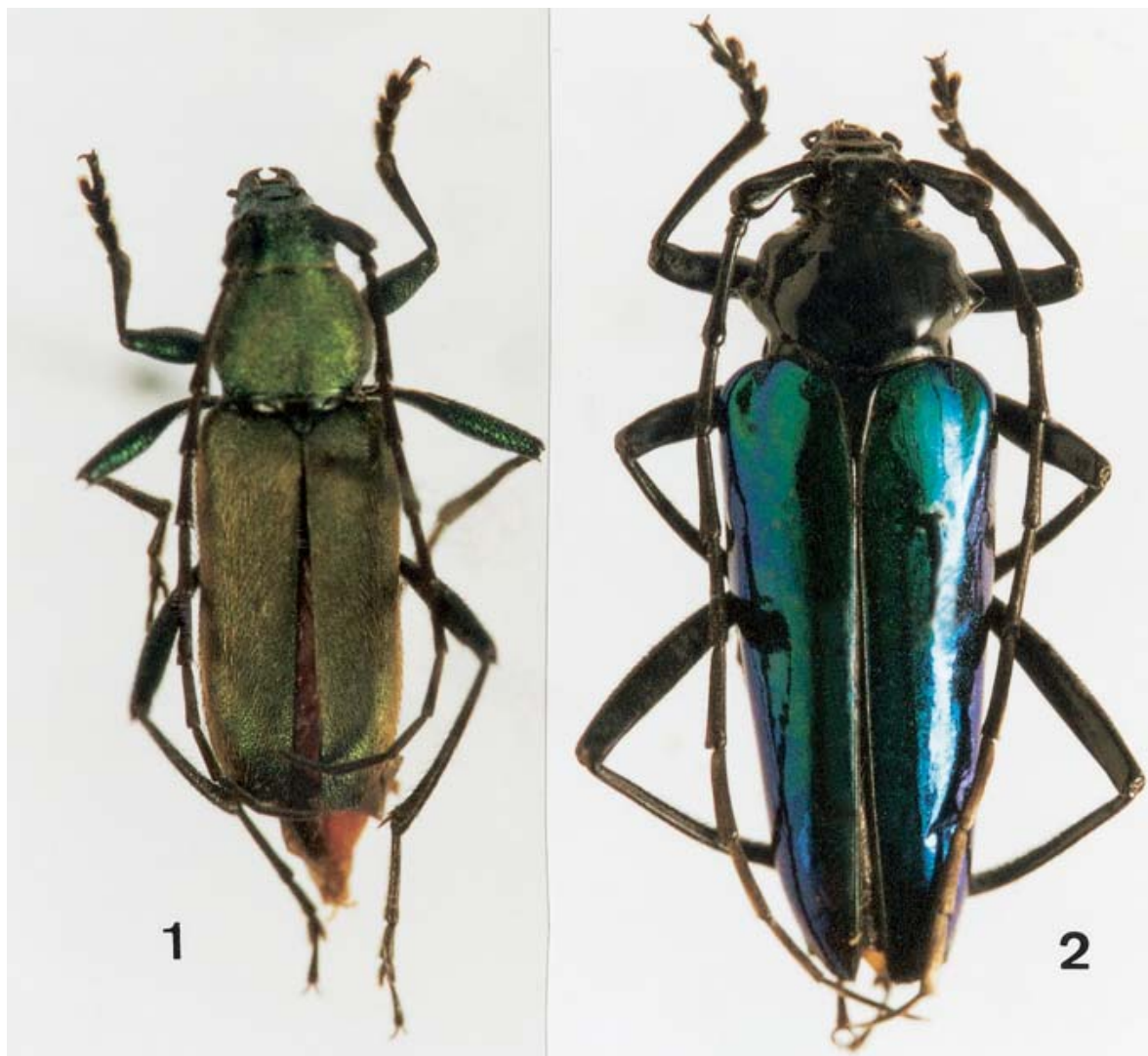

Figs. 1-2. 1, Chrysoprasis pilosa sp. nov., holótipo -, comprimento 9,4 $\mathrm{mm}$; 2, Crioprosopus cacicus Bates, -, comprimento 26,0 mm.

em toda a superfície. Partes laterais do protórax com mesmo tipo de pontuação e pilosidade apresentadas no dorso. Élitros densamente pontuados e microesculturados; distância entre os pontos menor que o comprimento das cerdas, que são abundantes e amarelodouradas. Quando a cabeça está voltada para a fonte luminosa, as cerdas recobrem toda a declividade lateral. Extremidades elitrais obliquamente truncadas com dentículo no lado externo. Lados do metasterno fina e densamente pontuados ântero-lateralmente; restante da superfície com pontos grossos: o diâmetro dos pontos maior que a distância entre os pontos. Dois terços apicais dos fêmures com forte brilho metálico; extremidades dos metafêmures com as abas apicais aguçadas e densamente pontuados no lado externo.

Dimensões, em mm, C Comprimento total, 9,4; protórax: comprimento 2,1; maior largura, 2,6; comprimento do élitro, 6,5; largura umeral, 3,0.

Material-tipo. COLÔMBIA, Huila: Garzon $\left(2^{\circ} 12^{\prime} 03^{\prime \prime N}, 75^{\circ} 38^{\prime} 25^{\prime \prime} \mathrm{W}, 828 \mathrm{~m}\right)$, holótipo ‘, 10.V.1971, F. Ramírez col., "potrero maleza" (UNAB).

Discussão. Seguindo-se pela chave de NAPP \& MARTINS (1998), C. pilosa apresenta: (1) antenas com espinho desenvolvidos nos antenômeros III-V (VI); (2) metasterno com 
pontuação grossa; (3) meso- e metasterno com colorido verde-metálico a verde-douradometálico; (4) metafêmures densamente pontuados e (5) protórax mais largo do que longo. Esses caracteres conduzem a Chrysoprasis hypocrita Erichson, 1847. Chrysoprasis pilosa difere de C. hypocrita principalmente pela pilosidade amarelo-dourada do pronoto e dos élitros. Em C. hypocrita, o pronoto e élitros têm pêlos curtos e pouco aparentes.

\section{Trachyderini, Ancylocerina \\ Ceralocyna variegata Monné \& Napp, 2000}

Ceralocyna variegata MonNÉ \& NAPP, 1999:35, fig. 4; 2000:118, figs. 81, 82, 91.

O exemplar que atribuímos a esta espécie diverge em detalhes de colorido do materialtipo, um casal de Rondônia, Brasil. Neste macho, os úmeros não são pretos, mas concolores com o terço basal avermelhado; o metasterno é avermelhado e preto somente na borda anterior e o terço basal dos meso- e metafêmures é preto com apenas estreitíssima porção da base mais avermelhada. Os caracteres morfológicos coincidem com a descrição original, porém este registro deve ser considerado com reservas até que mais espécimens possam ser examinados.

Material examinado. COLÔMBIA, Cundinamarca: Cachypay $\left(5^{\circ} 16^{\prime} 22^{\prime \prime N}, 74^{\circ} 34^{\prime} 22^{\prime \prime} \mathrm{W}\right)$, ‘, 2.IV.1972, A. Martínez col., "en suelo" (UNAB).

\section{Trachyderina \\ Crioprosopus cacicus Bates, 1885}

(Fig. 2)

Crioprosopus cacicus Bates, 1885:320; MonnÉ, 1994b:38 (cat.).

Após a descrição original esta espécie nunca mais foi mencionada, a não ser em catálogos, e esta é uma oportunidade para figurá-la (fig. 2). A coloração da face ventral do corpo preta, com áreas laterais no metasterno e parte dos urosternitos, exceto o V, com regiões avermelhadas coincide com a descrição original. A localidade-tipo é apenas Colômbia e apresentamos dado de ocorrência mais preciso.

Material examinado. COLÔMBIA, Boyacá: Coscuez, '-, 13.IV.1992, A. Cubillos R. col. (UNAB).

\section{Ceragenia insulana Fisher, 1943}

Ceragenia insulana Fisher, 1943:12; MonNé, 1994b:30 (cat.).

Originalmente descrita do Panamá, Canal Zone, Barro Colorado Island e figurada por Monné \& Martins (1991).

Material examinado. COLÔMBIA, Magdalena: PNN (Parque Nacional Natural) Tayrona (Cañaveral, $11^{\circ} 20^{\prime} \mathrm{N}, 7^{\circ} 02^{\prime} \mathrm{W}, 30 \mathrm{~m}$ ), $2^{\prime} \cdot$, 19-30.IX.2000, R. Henriquez col., armadilha Malaise (IAHC, MZSP).

\section{Ancylosternus morio morio (Fabricius, 1787)}

Cerambyx morio Fabricius, 1787:133.

Trachyderes morio; DaLman, 1817:365.

Ancylosternus morio; Aurivillius, 1912:481.

Ancylosternus morio morio; Huedepohl, 1985:88, figs. 55j, 57k; Monné, 1994b:93; Martínez, 2000:91.

Cerambyx scutellaris Olivier, 1795:67, est. 21, fig. 160.

Iheringia, Sér. Zool., Porto Alegre, 93(1):31-36, 30 de março de 2003 
Ancylosternus scutellaris; Dupont, 1834:50; Huedepohl, 1985:88 (sin.)

Trachyderes (Ancylosternus) scutellaris; LAPORTE, 1840:418.

HuEDEPOHL (1985) distinguiu as subespécies pelos ápices dos élitros (ângulos denteados em A. m. morio e ângulos espinhosos em A. morio albicornis Erichson, 1847) e tubérculo prosternal (elevado em A. m. morio e pequeno, indistinto, em A. morio albicornis). Ancylosternus m. morio ocorre na Colômbia (Antióquia, Quindío), Venezuela (Aragua, Zulia, Sucre, Distrito Federal) e Guiana Francesa e A. morio albicornis, no Peru, Brasil (Mato Grosso e São Paulo) e Bolívia. MARTínez (2000) registrou-a para Antióquia, Bolívar, Cesar, Cundinamarca e Magdalena.

Material examinado. COLÔMBIA, Santander: Barichara (6³8’20"N, 73¹3’38"W, 1294 m), ‘’, 6.VI.1989, O. Higuera \& R. Quesada col. (UNAB).

\section{Ozodera callidioides Dupont, 1840}

Ozodera callidioides Dupont, 1840b:42; MonnÉ, 1994b:84 (cat.).

Ozodera xanthospilos Dupont, 1840a:est. 37; Huedepohl, 1985:21 (sin.).

Ozodera bipartita BuQuet, 1840:111; HuedePOHL, 1985:21 (sin.).

Ozodera callidioides e seus sinônimos foram originalmente descritos da Colômbia, sem especificação maior, exceto $O$. bipartita registrada para Bogotá.

Material examinado. COLÔMBIA, Cundinamarca: Bogotá (Cerros Ubaquen), ‘., 27.VIII.1989, M. Corradine col. (GMIC).

\section{Trachyderes hermani Huedepohl, 1985}

Trachyderes hermani Huedepohl, 1985:104, figs. 48, 56c; Monné, 1994b:96 (cat.); Martínez, 2000:92.

Foi assinalada por MARTínEZ (2000) para Santander, com base apenas na localidade-tipo.

Material examinado. COLÔMBIA, Boyacá: San Luis de Gaceno $\left(4^{\circ} 49^{\prime} 21^{\prime \prime} \mathrm{N}, 63^{\circ} 10^{\prime} 13^{\prime \prime} \mathrm{W}\right.$, $400 \mathrm{~m}$ ), ‘', 8.III.1992, J. Rueda col. (UNAB).

\section{Aegoidus debauvei (Guérin-Méneville, 1838)}

Phoedinus debauvei Guérin-MÉNEviLle, 1838:287.

Trachyderes venustus Newman, 1840:3; White, 1853:66 (sin.).

Aegoidus debauvei; White, 1853:66; Monné, 1994b:81 (cat.); Martínez, 2000:91 .

Monné (1994b) assinalou A. debauvei para o Panamá, Colômbia, Guiana e Brasil (Roraima). MARTínEz (2000) registrou-a para Santander, Colômbia.

Material examinado. COLỔMBIA, Meta: Puerto López $\left(4^{\circ} 05^{\prime} \mathrm{N}, 72^{\circ} 58^{\prime} \mathrm{W}, 181 \mathrm{~m}\right)$, ‘', 3.IX.1972, Salazar col. (MZSP); '-, IX.1974, V. Velásquez col. (UNAB).

Agradecimentos. A Antonio Santos Silva (MZSP) pela execução das fotografias. O material de IAHC foi colecionado com auxílio concedido a Michael Sharkey e Brian Brown pelo National Science Foundation, USA (DEP9972024).

\section{REFERÊNCIAS BIBLIOGRÁFICAS}

Aurivillius, C. 1912. Coleopterorum Catalogus, pars 39, Cerambycinae. Berlin, W. Junk. 574 p. Bates, H. W. 1885. Biologia Centrali-Americana. Supplement to Longicornia. London, British Museum (Natural History). v.5, p.249-426, est.17-24.

Blackwelder, R. E. 1946. Checklist of the coleopterous insects of Mexico, Central America, the West Indies and South America. Part 4. Bull. U. S. natn. Mus., Washington, 185:551-763. 
Buquet, A. 1840. Description d'une nouvelle espèce de trachyderide appartenent au genre Ozodera. Revue Zool., Paris, 1840:110-112.

Chevrolat, A. 1862. Révision des genres Eriphis et Mallosoma Serville, du groupe des clytides et description de trois nouveaux genres dont un doit etre rapporté au groupe des callidiites. Annls Soc. ent. Fr., Paris, (4)2:747-763.

Dalman, J. W. 1817. Appendix ad C. J. Schoenherr. In: Schoenherr, C. J. Synonymia Insectorum... Skaris, Lewerentzichen Buch. v. 1, n. 3, 506p.

Dupont, H. 1834. In: Audinet-Serville, J. G. Nouvelle classification de la famille des longicornes. Annls Soc. ent. Fr., Paris, Sér. 1, 3:5-110.

1840a. Supplement à la monographie des trachydérides. Mag. Zool., Paris, 1840, est. 28-38.

-. 1840b. Nouvelle espèce du genre Ozodera. Revue Zool., Paris, 1840:42.

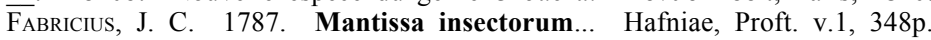

Fischer, W. S. 1943. A new cerambycid beetle from the Canal Zone. Boln Dep. Sanid. Veget., Santiago, 3(1):12-14.

Giesbert, E. F. \& Chemsak, J. A. 1997. A review of the genus Euderces Le Conte (Tillomorphini). Proc. Calif. Acad. Sci., San Francisco, 49(8):211-286.

Gounelle, E. 1913. Cérambycides nouveaux de Colombie, appartenent au Musée de Hambourg (Col.). $2^{e}$ note. Bull. Mus. natn. Hist. nat., Paris, 1913:193-231.

Guérin-Méneville, F. E. 1838. Note sur deux espèces nouvelles du genre Phoedinus. Revue Zool., Paris, 1838:286-287.

Huedepohl, K.-E. 1985. Revision der Trachyderini (Coleoptera, Cerambycidae, Cerambycinae). Ent. Arb. Mus. G. Frey, Tutzing bei München, 33/34:1-167.

Lacordaire, J. T. 1869. Genera de Coléoptères... Paris, Roret. v. 8, 552 p.

LAPORTE, F. L. N. 1840. Histoire Naturelle des Insectes Coléoptères. Paris, P. Duméril. v.2, 563p., 36 est.

Martínez, C. 2000. Escarabajos longicórnios (Coleoptera: Cerambycidae) de Colômbia. Biota Colômbiana, Bogotá, 1(1):76-105.

Martins, U. R. \& Galileo, M. H. M. 2002a. Cerambycidae (Coleoptera) da Colômbia. I. Eburiini (Cerambycinae). Iheringia, Sér. Zool., Porto Alegre, 92(4):5-10.

. 2002b. Cerambycidae (Coleoptera) da Colômbia. II. Ibidionini (Cerambycinae). Iheringia, Sér. Zool., Porto Alegre, 92(4):11-18.

Monné, M. A. 1993. Catalogue of the Cerambycidae (Coleoptera) of the western hemisphere. Part IX. São Paulo, Sociedade Brasileira de Entomologia. 131 p.

. 1994a. Catalogue of the Cerambycidae (Coleoptera) of the western hemisphere. Part X. São Paulo, Sociedade Brasileira de Entomologia. 81 p.

. 1994b. Catalogue of the Cerambycidae (Coleoptera) of the western hemisphere. Part XI. São Paulo, Sociedade Brasileira de Entomologia. 157 p.

Monné, M. A. \& Martins, U. R. 1991. Sinopse do gênero Ceragenia Audinet-Serville, 1834 (Trachyderini). Revta bras. Ent., São Paulo, 35(1):197-201.

Monné, M. L. \& NAPP, D. S. 1999. Ceralocyna (Coleoptera, Cerambycidae): descrição de quatro espécies novas e sinonímia. Iheringia, Sér. Zool., Porto Alegre, (86):29-37.

2000. Revisão do gênero Ceralocyna (Coleoptera, Cerambycidae, Cerambycinae). Iheringia, Sér. Zool., Porto Alegre, (88):103-137.

NAPp, D. S. \& Martins, U. R. 1998. Revisão do gênero Chrysoprasis A.-Serville, 1834 (Coleoptera, Cerambycidae). IV. Grupo hypocrita. Revta bras. Ent., São Paulo, 41(2-4):465-499.

Napp, D. S. \& Santos, B. B. Dos. 1999. Description of Monnecles, new genus, and redescription of Heterops loreyi Duponchel (Heteropsini). Coleop. Bull., Lawrence, 53(3):281-286.

Newmann, E. 1840. Entomological notes. Entomologist, London, 1:1-16.

Olivier, A. G. 1795. Entomologie, ou Histoire Naturelle des Insectes, avec leurs caractères génériques et spécifiques, leur description, leur synonymie et leur figure illuminée. Coléoptères. Paris, Lanneau. v.4, p. 519, 72 est.

Pascoe, F. P. 1866. List of the longicornia collected by the late Mr. P. Boucherd at Santa Marta.

Trans. ent. Soc. London, London, 1866:279-296, est. 20.

White, A. 1853. Catalogue of the coleopterous insects in the collection of the British

Museum. Longicornia 1. London, British Museum (Natural History). v.7, 174p., est.1-4.

Recebido em 30.04.2002; aceito em 02.09.2002.

Iheringia, Sér. Zool., Porto Alegre, 93(1):31-36, 30 de março de 2003 